\title{
Comparisons of Center of Mass during Golf Swings of 7 Iron or Driver
}

\author{
Sukwon Kim \\ Department of Physical Education, Jeonbuk National University, Jeonju, Jellabuk-do, South Korea \\ Correspondence should be addressed to Sukwon Kim; rockwall@jbnu.ac.kr
}

Copyright $\odot 2022$ Made Sukwon Kim. This is an open-access article distributed under the Creative Commons Attribution License, which permits unrestricted use, distribution, and reproduction in any medium, provided the original work is properly cited.

\begin{abstract}
Golf game is competitive and it has attracted many participants due to its unique features. In the many features, kinetics of golf swing was analyzed to improve one's performance level. The present study examined the center of mass (COM) of a recreational golfer during a phase from address to backswing top for two different golf clubs. Kinematic data were evaluated. The results indicated that there were some differences in COM between two different golf clubs.
\end{abstract}

KEYWORDS- COM, driver, golf, swing, 7-iron.

\section{INTRODUCTION}

The golf swing is the most important part of a game of golf. Therefore, the scientific research and motion analysis should benefit trainers and golfers to develop and learn theoretical basis and guidance for training and teaching $[1,2,3]$. Although a golf club is a relatively light object, when moving fast, it feels heavier providing instant feedback. A greater golfer should have ability to move the club freely, and has a steadier rhythm of the upper and lower body and, thus, demonstrates a better performance $[4,5,6,7]$. The greater golfer can maintain a better balance during the swing and his/her body should remain free to swing without interference from the club and must maintain a balanced posture during the swing $[7,8]$. The driver is the most important club because the current golf game requires players to drive a ball longer, and, also, the 7-iron are used most frequently in the practice range. The driver is widely recognized by golfers as the most difficult club for consistent accuracy $[9,10]$ because of the longer shaft, larger head, smaller loft, lighter weight and larger range of motion. The 7-iron is a beginner's club and the shaft length is right in the middle amongst all clubs. The purpose of the present study was to examine COM during "address-to-backswing top" phases of 7-iron and driver of a recreational golfer.

\section{METHOD}

\section{A. Participants}

One recreational golfer participated in the present study. He had no history of upper and lower extremity injuries and no physical training within 24 hours. The subject was informed with the purpose of the experiment, the procedure, and signed a consent form. A motion capture system Motive Body2.2.0 and 4 OptiTrack 17W cameras (NaturalPoint, Inc, Corvallis, OR USA), were used for motion data collection. This system can capture and record 3D movements precisely.

\section{B. Procedure}

The subject was asked to perform golf swings with a 7-iron or a driver. He used his own golf clubs. For the purpose of the present study, only a phase from address to backswing top was analyzed (figure 1)
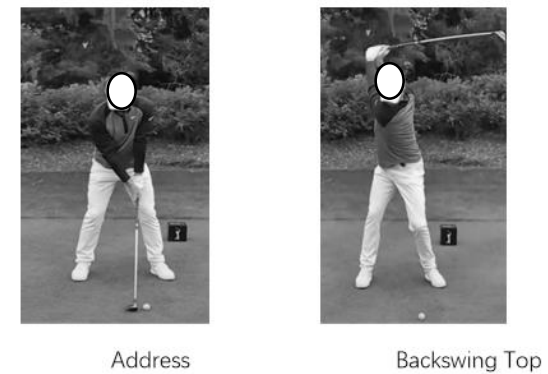

Figure 1. Golf Swing from Address to Backswing Top

\section{RESULTS}

At the moment of address, the golfer uses the driver with the center of mass positioned more to the right side of the body in the coronal plane, more to the back side of the body in the sagittal plane, and higher in vertical height than the 7 club (Figure $2 \& 3$ ). The player's center of mass trajectory is consistent throughout the backswing. With different clubs, the player's center of mass was closer to the coronal and height positions at the top of the backswing(Figure 3). 


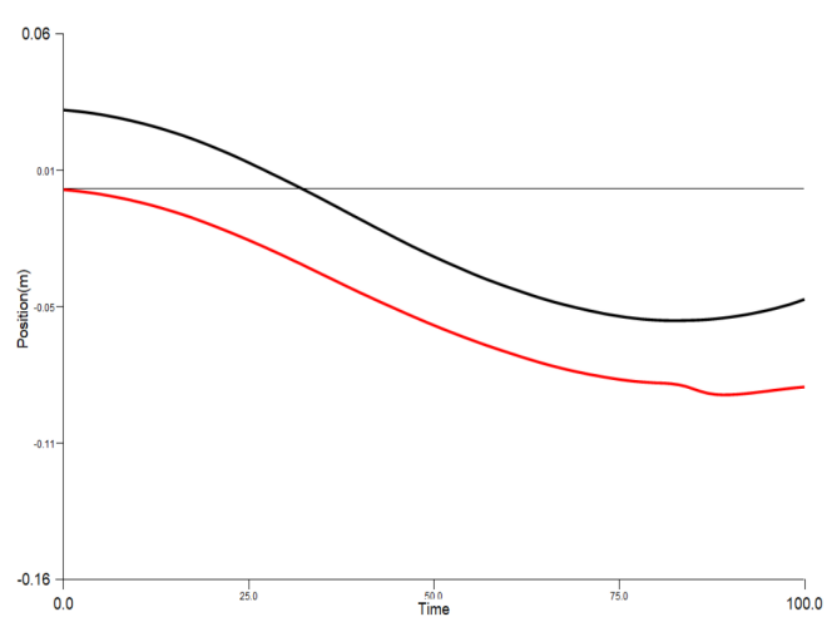

Figure 2: COM trajectory (medial-lateral axis) from Address to Backswing Top

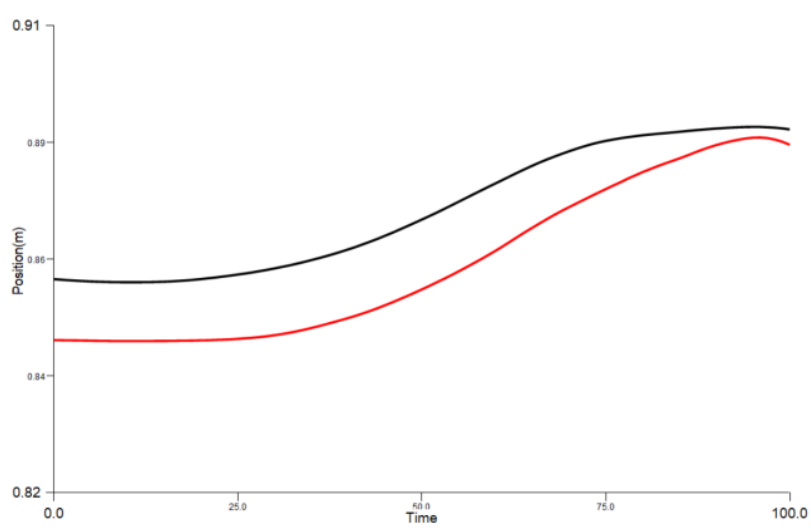

Figure 3: COM trajectory (vertical axis) from Address to Backswing Top

\section{DISCUSSION}

The study objective was to examine COM of a recreational golfer during swing of 7 iron or driver.

Center of mass is the point in a body or system of bodies at which, for certain purposes, the entire mass may be assumed to be concentrated. The force acting on the center of mass is equal to all the forces acting on the system moving in parallel to this point.

The displacement and velocity of the COM is meaningful variables that allow evaluation of dynamic balance ability according to the skill level of golfers $[11,12,13,14]$. From the top of the backswing, the golfer's center of mass tends to move downward and below the height of the start position [10]. Smaller change of center of mass from the top of backswing to hit the ball moment, means that the more stable the golfer's center of mass is during the downswing, and the smaller the effect on the hitting effect $[10,11,14,15]$

The rotation generated by the body during the downswing transfers the force continuously from the feet to the club head $[13,14,15]$. The power generated in the lower body is transferred through the hips to the trunk, then from the trunk to the shoulders, then from the shoulders to the arms, then from the hands and shoulders to the wrists and hands, and then to the club head. From the perspective of sports biomechanics, golf is a rotational movement along the longitudinal axis of the human body $[7,11,14,15]$. The golf swing technique is a movement that rotates around the longitudinal axis of the body; this longitudinal axis passes from the head to the center of the body, and with this axis as the center, the club, shoulders, waist and lower limbs are swung with two arms and two hands to make a gyration, and the swing trajectory should be a relatively uniform large arc $[14,15,16]$.

\section{CONCLUSION}

The study concluded that there were some differences in COM during golf swings of two different clubs.

\section{CONFLICTS OF INTEREST}

The authors declare that they have no conflicts of interest.

\section{ACKNOWLEDGEMENT}

This research was supported by "Research Base Construction Fund Support Program" funded by Jeonbuk National University in 2021 2022.

\section{REFERENCES}

[1] Stauch M, Liu Y, Giesler M, Lehmann M. Physical activity level during a round of golf on a hilly course. J Sports Med Phys Fitness. 1999 Dec;39(4):321-7. PMID: 10726433.

[2] Luscombe J, Murray AD, Jenkins E, Archibald D. A rapid review to identify physical activity accrued while playing golf. BMJ Open. 2017 Nov 28;7(11):e018993. doi: $\quad 10.1136 /$ bmjopen-2017-018993. PMID: 29187418 ; PMCID: PMC5719314.

[3] Zunzer SC, von Duvillard SP, Tschakert G, Mangus B, Hofmann P. Energy expenditure and sex differences of golf playing. J Sports Sci. 2013;31(10):1045-53. doi: 10.1080/02640414.2013.764465. Epub 2013 Jan 30. PMID: 23362842.

[4] Broman G, Johnsson L, Kaijser L. Golf: a high intensity interval activity for elderly men. Aging Clin Exp Res. 2004 Oct;16(5):375-81. doi: 10.1007/BF03324567. PMID: 15636463

[5] Murray AD, Daines L, Archibald D, Hawkes RA, Schiphorst C, Kelly P, Grant L, Mutrie N. The relationships between golf and health: a scoping review. Br J Sports Med. 2017 Jan;51(1):12-19.

[6] Gluck GS, Bendo JA, Spivak JM. The lumbar spine and low back pain in golf: a literature review of swing biomechanics and injury prevention. Spine J. 2008 Sep-Oct;8(5):778-88.

[7] Cole MH, Grimshaw PN. The Biomechanics of the Modern Golf Swing: Implications for Lower Back Injuries. Sports Med. 2016 Mar;46(3):339-51.

[8] Choi A, Joo SB, Oh E, Mun JH. Kinematic evaluation of movement smoothness in golf: relationship between the normalized jerk cost of body joints and the clubhead. Biomed Eng Online. 2014 Feb 26;13(1):20.

[9] Horan SA, Evans K, Kavanagh JJ. Movement variability in the golf swing of male and female skilled \begin{tabular}{lllll} 
golfers. Med Sci & Sports Exerc. 2011 \\
\hline
\end{tabular} 
Aug;43(8):1474-83.

[10] Langdown BL, Bridge M, Li FX. Movement variability in the golf swing. Sports Biomech. 2012 Jun;11(2):273-87.

[11] Morrison A, McGrath D, Wallace ES. The relationship between the golf swing plane and ball impact characteristics using trajectory ellipse fitting. J Sports Sci. 2018 Feb;36(3):303-310.

[12] Worobets J, Stefanyshyn D. The influence of golf club shaft stiffness on clubhead kinematics at ball impact. Sports Biomech. 2012 Jun;11(2):239-48.

[13] MacKenzie SJ, Boucher DE. The influence of golf shaft stiffness on grip and clubhead kinematics. J Sports Sci. 2017 Jan;35(2):105-111.

[14] Otto SR. Some applications of mathematics in golf. Proc Math Phys Eng Sci. 2017 Aug;473(2204):20170392.

[15] Adlington GS. Proper swing technique and biomechanics of golf. Clin Sports Med. 1996 Jan;15(1):9-26.

[16] Selicki FA, Segall E. The mind/body connection of the golf swing. Clin Sports Med. 1996 Jan;15(1):191-201. 